\title{
Non-Destructive Inspection System for Welding Processes by applying Data Fusion
}

\author{
by K. Simmen*, B. Buch*, A. Breitbarth*, G. Notni*,** \\ * Technische Universität IImenau, Group for Quality Assurance and Industrial Image Processing, Gustav- \\ Kirchhoff-Platz 2, 98693, IImenau, Germany, Katharina.Simmen@tu-ilmenau.de \\ ${ }^{* *}$ Fraunhofer Institute for Applied Optics and Precision Engineering IOF, Albert-Einstein-Str. 7, 07745, Jena, \\ Germany
}

\section{Abstract}

Welding processes are influenced by many parameters. By applying multimodal data fusion of multichannel image data and signals of various welding parameter, the correlation of these and their influence on weld seam imperfections are investigated. The application of a multispectral area camera array enables a new approach on non-destructive testing particularly supported by the passive thermography. The results show the different cooling behaviour of several welding imperfections, such as porosity, and present the relation between incorrect welding parameters and imperfections.

\section{Introduction}

Automatic welding is state of the art in many areas of metal working, such as automotive industry. These highly automated manufacturing processes mainly rely on computer vision. The supervision of welding processes by using inline monitoring equipment shall reduce time consuming and expensive reworking. Existing monitoring systems apply laser sensors for on-the-fly seam tracking on fillet welds at laser welding processes [1]. There are also arc welding systems that use triangulation sensors to contactlessly scan the weld seam [2]. These monitoring systems work with light section, which allows the measurement of a material edge along a projected line of light. This information is used exclusively to guide the welding torch along the determined edges. The weld seams are not inspected for imperfections. Another monitoring system also works with light section sensors but uses this technology for two- and three-dimensional weld-seam verification [3]. However, defect detection only takes place after completion of the welding process. Therefore, this information only has a delayed effect on the production process. Systems for detection of imperfections by using line scan cameras are used in the production of such as endless welded tubes. These are devices for evaluating the quality of welds by measuring the temperature profile of the cool down melting during the welding process [4]. These system works in a narrow band of infrared spectrum.

However, in small-batch and single-item production such as in heavy steel construction (bridges, lock-gates) semi-automatic manufacturing systems are mainly established, with the majority of production being carried out by hand. Furthermore, the quality of the welding seams is assessed exclusively by visual inspection (DIN EN 13018 and DIN EN ISO 5817) by qualified employees as an additional step in the production process. Increasing requirements for quality assurance and production monitoring lead to rising quantity of weld seams requiring inspection. The use of automated inline systems for non-destructive testing (NDT) of high-volume steel constructions is a promising approach and a significant economic driving force for constructive steel engineering in single-item production. Process automation for weld seam inspection greatly increases productivity. Thereby, an additional working step can be avoided. By using thermography, a demand-actuated NDT can be implemented. Finally, early recognition of weld imperfections and increasing the automation level of manufacturing processes leads to a quality and productivity enhancement.

Within the scope of a project, a robot-controlled manufacturing system is to be developed and used to eliminate the effect due to the physical fatigue of the worker and to ensure a consistent quality of the end product. The development of an optical welding inspection system introduces preparatory tasks. For example, the selection of appropriate cameras, the correction of perspective-based distortion of the images and a suitable experimental setup. Especially, the exclusive analysis of fillet welds and the associated alignment of the steel plates in a T-shape complicate these tasks. This weld seam shape was chosen because it is the most common in steel construction. [5]

\section{Passive Thermography for non-destructive Inspection and Data Fusion}

In this paper an inspection system for welding processes supported by a combined set of cameras (multispectral and infrared) will be introduced. Due to the optical monitoring system a non-contact system for non-destructive inspection has been enabled. The use of cameras with different sensitivities provides a wide spectral bandwidth from the visual to the far infrared wavelength range. The passive thermography with the long wave infrared camera enhances the system to an in-line capable monitoring system. To detect defects immediately after they occur, this inspection system is used during the process and can therefore be regarded as a real-time system at low welding speeds of up to approximately $50 \mathrm{~cm} / \mathrm{min}$.

The idea of using data fusion is the combination of information from different sources: cameras with different spectral ranges as well as data sets of welding parameters. The imperfections that occur during the welding process but may remain undetected during the subsequent visual inspection can be documented by this system and contribute to 
quality assurance. Finally, it can be summarized that this monitoring system leads to an extensive digital copy of the welding seam and process, which can be extended by means of further sensors and new knowledge in the interpretation of the data.

\section{Experimental Setup}

\subsection{Measuring Instrumentation and Measuring Process}

For the experiments, a camera array consisting of three cameras is used, each covering different spectral ranges, as shown in Fig. 1. The VIS- and the NIR-camera are multispectral mosaic snapshot cameras with multiple narrow band spectrum in visible or near infrared light, respectively. Both cameras have a total resolution of 2,048 $\times 1,088$ pixels. The detector is coated with a mosaic filter of 16 (VIS-camera) and 25 (NIR-camera) spectral frequency bands. The channel separation in narrow band images results in a spatial resolution of approximately $512 \times 272$ pixels for the VIS-camera and approximately $409 \times 217$ pixels for the NIR-camera. [6] The used lenses have a focal length of $16 \mathrm{~mm}$ and a minimum working distance of $10 \mathrm{~cm}$. Due to the coating for VIS and NIR from $\lambda=425$ to $1000 \mathrm{~nm}$ the application on both cameras is intended. The third camera within the array is a broadband LWIR-camera, which uses an un-cooled microbolometer focal plane detector array with a size of $1,024 \times 768$ pixels. The spectral sensitivity of the camera ranges from $7.5 \mu \mathrm{m}$ to $14 \mu \mathrm{m}$. The associated lens has a focal length of $30 \mathrm{~mm}$ and a minimum working distance of about $30 \mathrm{~cm}$. [7] The acquisition system records an image every $500 \mathrm{~ms}$.

VIS-camera

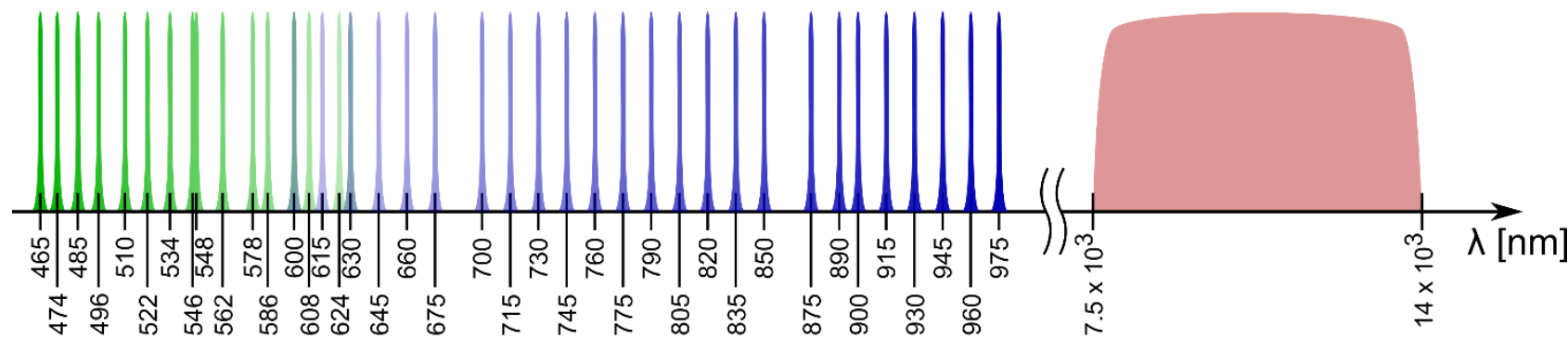

Fig. 1: Hyperspectral bands of the camera array; narrow bands of VIS- and NIR-camera, broadband of LWIRcamera

The VIS- and NIR-cameras are mounted to one side of the LWIR-camera (Fig. 2 a). All cameras are focused at a common focal point at a working distance of $30 \mathrm{~cm}$ (Fig. $2 \mathrm{~b}$ ). Due to this camera arrangement, the recorded images have almost identical image sections. Preliminary studies have revealed at the working distance of $30 \mathrm{~cm}$ a field of view (FOV) of $19.9 \times 10.6 \mathrm{~cm}(\mathrm{VIS} / \mathrm{NIR})$ and $18 \times 13 \mathrm{~cm}(\mathrm{LWIR})$. The FOV is indicated in horizontal and vertical dimensions. With this camera arrangement, the horizontal camera planes are at an angle of $12^{\circ}$ to each other. This alignment leads to a perspective-based distortion between the images of the different cameras and has to be corrected by projective transformation (see section 4.1).

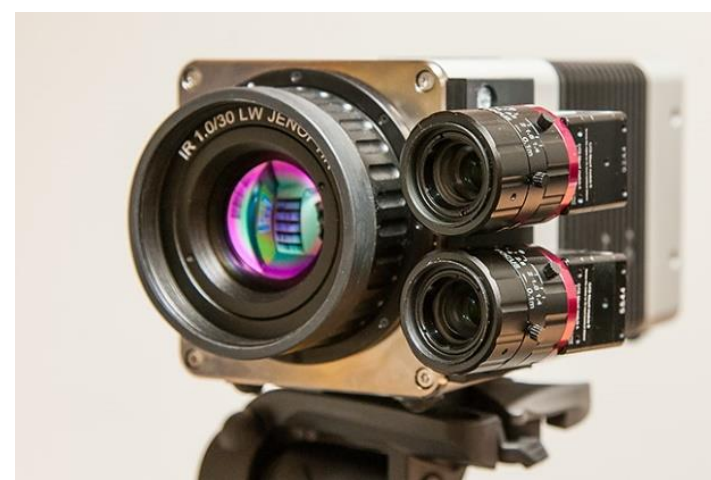

a)

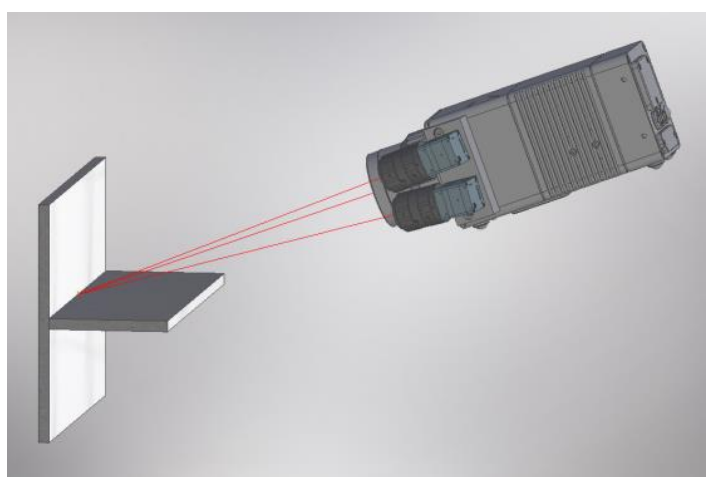

b)

Fig. 2: a) camera array (front view); b) measurement setup with $T$-shaped specimen, camera array and focal point

The acquisition process can be divided into three steps (pre-, in- and post-processing) and is a combination of inline and offline supervision of the welding process. The pre-processing step captures the initial situation for the evaluation with the results of the post-processing. Measurements of surface characteristics are performed using the VIS- and NIR- 
camera. For base material with a thickness of more than $25 \mathrm{~mm}$, the LWIR-camera records the heat distribution after the preheating event of this material. [8] The in-line process monitoring is the centrepiece of the process and enables the observation of the temperature distribution of the weld seam during the welding process. Especially, the changes occurring a few seconds after the welding event can be observed, such as the influence of the aggregate state of the material on the emissivity. Within this step only the LWIR-camera is active because the other cameras are overexposed by the arc. During the last step immediately after the end of the welding process, all three cameras observe the completed weld seam. For the evaluation of the weld seam the measurement by the NIR-camera will be continued. The primary goal of this is to determine the cooling process on the basis of PLANCK's law of black body radiation. Finally, the main objective is the recording of all these process-relevant data to deduce control parameters in order to improve the welding process. [9]

\subsection{Manufacturing Process and Welding Conditions}

The investigated welding scenario is a one-side fillet weld in a tilted T-shape (Fig. $3 \mathrm{a}$ ). As material for the steel parts S355 will be applied. Two plates with a size of $100 \times 150 \times 5 \mathrm{~mm}^{3}$ are welded with weld thickness $a=3 \mathrm{~mm}$ and weld seam length of approximately $80 \mathrm{~mm}$, as presented in Fig. $3 \mathrm{a}$ ). The welding direction is along the positive $y$-axis, as shown by the red arrow in Fig. $3 \mathrm{~b}$ ). The fillet welds are performed by a 6 -axis anthropomorphic robot to ensure the repeatability and process stability.

In the experiments, the MAG welding process is used to create the welding seams. The wire diameter $d_{w}=1 \mathrm{~mm}$, the wire material G3SI1, the wire feed speed $v_{f}=7 \mathrm{~m} / \mathrm{min}$, the welding speed $v_{w}=25 \mathrm{~cm} / \mathrm{min}$ and shielding gas composition $98 \% \mathrm{Ar} / 2 \% \mathrm{CO}_{2}$ are fixed parameters in the manufacturing process. The flow rate of protective gas will be changed systematically in the particular experiment to reproduce defined imperfections. Therefore, the welding current $I$ and welding voltage $U$ are oscillating parameters because of their dependence on the stability of the process. This influence will be shown by the standard deviation in the results in section 5 .

The camera array described above is positioned at right angles to the weld and will be placed approximately $30^{\circ}$ above the $x y$-plane. The working distance of $30 \mathrm{~cm}$ to the T-shape have to be maintained to ensure the position of the focal plane in front of the specimen. The array is static to the device under test (DUT) and only the welding torch is moved by the robot. [9]

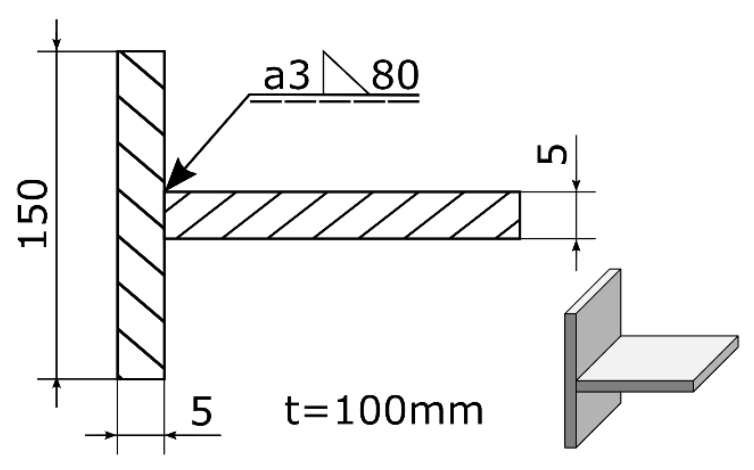

a)

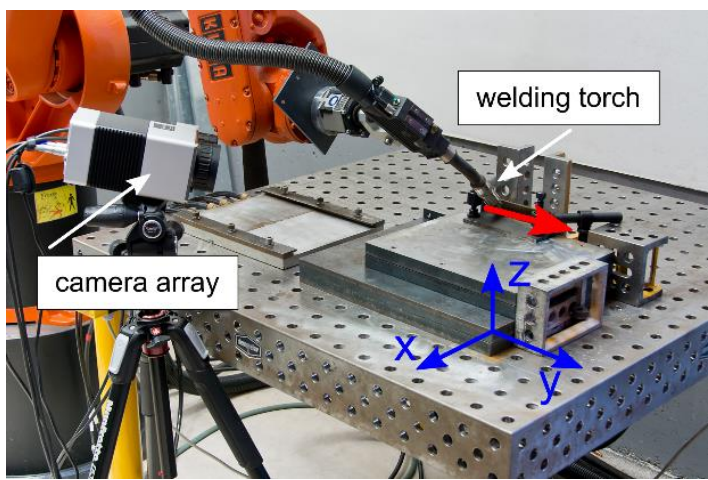

b)

Fig. 3: a) Geometry of the T-shape through a fillet weld, dimension in picture depth: $t=100 \mathrm{~mm}$; b) Manufacturing and measurement setup for fillet welds, including welding direction (red arrow)

\section{Projective Transformation and Digital Image Processing}

\subsection{Perspective-based Distortion}

The image acquisition by three different cameras with three different resolutions and three different alignments to the DUT leads to a perspective-based distortion. In order to make the images comparable, every single image must be rectified in relation to a reference image. Distorted images can be distorted in perspective (such as translation, rotation and scaling) with by means of projective transformation. This transformation is defined as a relationship between points of two images: distorted image $\boldsymbol{x}$ and undistorted image $\boldsymbol{x}^{\prime}$.

$$
\boldsymbol{x}=\boldsymbol{T}\left(\boldsymbol{x}^{\prime}\right) \text { or } \boldsymbol{x}^{\prime}=\boldsymbol{T}^{-1}(\boldsymbol{x})
$$

There are a forward and a backward transformation as shown in equation (1), where $\boldsymbol{T}$ is the transformation matrix and $\boldsymbol{T}^{-1}$ the inverse transformation matrix. For the forward transformation the coordinates of the source image $\boldsymbol{x}$ are specified as a function of the input coordinates $\boldsymbol{x}^{\prime}$ or for backward transformation vice versa. [10]

The rectification is usually limited to the single image case with known control points of a calibration target. The used rectification pattern applied four circles in the arrangement as shown in Fig. 4. Computing the two-dimensional transformation matrix requires a rectification pattern with four pairwise linearly independent vectors. [11] This pattern must be recorded simultaneously by all used cameras. The difficulty with the LWIR-camera is to create a contrast that clearly 
separates the circles from the background. The use of a uniformly cooled or heated PCB plate with the calibration pattern is sufficient to produce the required thermal contrast. The optical contrast between the base material and the copper circles is sufficient for the VIS- and NIR-camera.

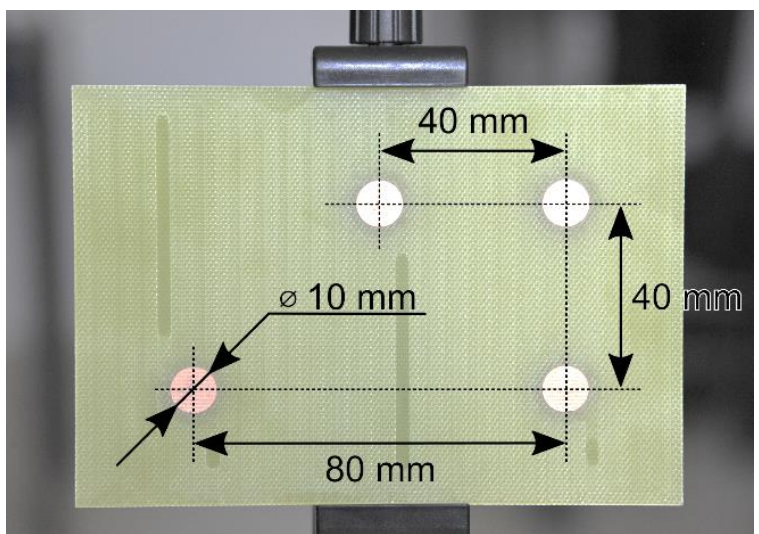

Fig. 4: PCB plate with rectification pattern, rotationally invariant with circles and its dimensions (black)

For the computing of the projective transformation the dimensions of the rectification pattern in each image have to be detected. By using circles, the estimation of diameters and centres can be achieved by the Hough transformation. This algorithm only works for images where the objects on the rectification pattern has a circular shape. In the described camera arrangement, there is only a slight deviation from the optical axis of the reference camera (LWIR camera). If a higher accuracy is required or a non-circular object shape is to be detected, the research of high precision edge probing by subpixel accurate edge position determination must be considered. [12]

The centres of the circles, named $\left(x_{1}, y_{1}\right)$ through $\left(x_{4}, y_{4}\right)$, in the source image are used to solve the following system of linear equations (Eq. 2), where $\lambda, \mu$ and $\tau$ are transformation coefficients. The matrix $S$ maps from the basis vectors $((1,0,0),(0,1,0),(0,0,1))$ to a multiple of the centre points $\left(x_{n}, y_{n}, 1\right)$ for $n=1,2,3$ and $(1,1,1)$ to $\left(x_{4}, y_{4}, 1\right)$. The matrix $\boldsymbol{S}$ is a map from basis vectors to the specified positions in the image.

$$
\begin{gathered}
\left(\begin{array}{ccc}
x_{1} & x_{2} & x_{3} \\
y_{1} & y_{2} & y_{3} \\
1 & 1 & 1
\end{array}\right) \cdot\left(\begin{array}{l}
\lambda \\
\mu \\
\tau
\end{array}\right)=\left(\begin{array}{c}
x_{4} \\
y_{4} \\
1
\end{array}\right) \\
\left(\begin{array}{ccc}
x_{1} & x_{2} & x_{3} \\
y_{1} & y_{2} & y_{3} \\
1 & 1 & 1
\end{array}\right) \cdot\left(\begin{array}{c}
x_{4} \\
y_{4} \\
1
\end{array}\right)=\left(\begin{array}{l}
\lambda \\
\mu \\
\tau
\end{array}\right) \\
\boldsymbol{S}=\left(\begin{array}{ccc}
\lambda \cdot x_{1} & \mu \cdot x_{2} & \tau \cdot x_{3} \\
\lambda \cdot y_{1} & \mu \cdot y_{2} & \tau \cdot y_{3} \\
\lambda & \mu & \tau
\end{array}\right)
\end{gathered}
$$

The columns represent all points in homogeneous coordinates by adding a 1 as the last entry. The representation in homogenous coordinates enables a uniform treatment of the transformations. The calculation of the map $\boldsymbol{R}$ of the reference image is done in the same way. The transformation matrix $\boldsymbol{T}$ is calculated using equation (3).

$$
T=R \cdot S^{-1}
$$

$\boldsymbol{T}$ is the combination map of the maps $\boldsymbol{S}$ and $\boldsymbol{R}$ and maps the source positions to the destination positions. $\boldsymbol{S}^{-1}$ maps from source positions to basis vectors, while $\boldsymbol{R}$ maps from there to destination positions. The rectification (Eq. 4 ) is realized by applying the transformation matrix $\boldsymbol{T}$ to all pixels in the source image $\boldsymbol{x}$.

$$
\left(\begin{array}{l}
x^{\prime} \\
y^{\prime} \\
z^{\prime}
\end{array}\right)=\boldsymbol{T} \cdot\left(\begin{array}{l}
x \\
y \\
1
\end{array}\right)
$$

The dehomogenization leads to the coordinate vector and thus to the positions in the destination image. The inhomogeneous coordinates are calculated by dividing by the third entry $z^{\prime}$ (Eq. 5).

$$
x^{\prime \prime}=\frac{x^{\prime}}{z^{\prime}} \text { and } y^{\prime \prime}=\frac{y \prime}{z^{\prime}}
$$

The rectification by projective transformation does not consider correction of the optical distortion (barrel and pincushion distortions) at the images. 


\subsection{Data Processing and Data Fusion}

The data acquisition system consists of different types of cameras and a recording device for the welding parameters. Initially, each data set is examined separately. Supported by a priori knowledge, image processing algorithms can be developed based on classical visual inspection.

The images of the LWIR camera clearly show a change in the emissivity for the advancing welding process. Especially, the shape of the edge between the different states of aggregation is characteristic. This change in emissivity can only be observed during the welding process. By determining the maxima and a minimum (Fig. 6 ) in each image along this edge, a appraisement can be made about the quality of the weld seam right during the process (see section 5).

Since the VIS-camera can only record before and after the welding process, examinations are carried out on the first and last image or their difference image, respectively. By convolution (Eq. 6) the difference image $A$ with the edge detection filter (Scharr operator: $\left.\boldsymbol{F}_{x}, \boldsymbol{F}_{y}\right)$, direction-independent gradient images $\left(\boldsymbol{G}_{x}\right.$ and $\left.\boldsymbol{G}_{y}\right)$ are generated.

$$
\boldsymbol{G}_{x}=\boldsymbol{F}_{\boldsymbol{x}} * A=\left[\begin{array}{ccc}
3 & 0 & -3 \\
10 & 0 & -10 \\
3 & 0 & -3
\end{array}\right] * A \quad \boldsymbol{G}_{\boldsymbol{y}}=\boldsymbol{F}_{\boldsymbol{y}} * A=\left[\begin{array}{ccc}
3 & 10 & 3 \\
0 & 0 & 0 \\
-3 & -10 & -3
\end{array}\right] * A
$$

An overall gradient image $\boldsymbol{G}$ is obtained by element-wise calculation with the following equation:

$$
G=\sqrt{G_{x}^{2}+G_{y}^{2}}
$$

Additionally, the direction of the gradient $\Theta$ is calculated via the atan2 function as shown in equation (8).

$$
\boldsymbol{\Theta}=\operatorname{atan} 2\left(G_{y}, G_{x}\right)
$$

The analysis of the incidence of angles occurring in the area of the weld seam allows conclusions to be drawn about its surface quality. The angles 0 or $-\pi$ and $\pi$ should be represented most frequently, as these characterize the edge of the weld to the base material. When inspecting a flawless weld seam, the values between $-\pi$ and 0 as well as 0 and $\pi$ should be weakly represented. The incidences at the values 0 and $\pi$ are added up to $p_{h}$. The variable $p_{v}$ represents the sum of the values at $-\frac{\pi}{2}$ and $\frac{\pi}{2}$. The ratio of $p_{v}$ and $p_{h}$ results in a value represents the amount of defects $a_{i}$ (Eq. 9) of each wavelength of the VIS-camera with $i=1 \ldots 16$.

$$
a_{i}=\frac{p_{v}}{p_{h}}
$$

Equation 10 shows $m$ the median of all amounts of defects and represents the average value of $a=\left(a_{1} \ldots a_{16}\right)$. The median is applied to reduce the influence of outliers.

$$
m=\operatorname{median}(\boldsymbol{a})
$$

Separately considered, all these methods are weak instruments for the evaluation of a weld seam. The fusion of the results from digital image processing and the data sets of the welding parameters provides a solid basis for evaluation of weld seams. The data fusion for the presented monitoring system is shown in Fig. 5. Based on a-priori knowledge, the fusion of different input variables leads to a substantiated result. On this basis a reliable decision about success or failure of the process can be made.

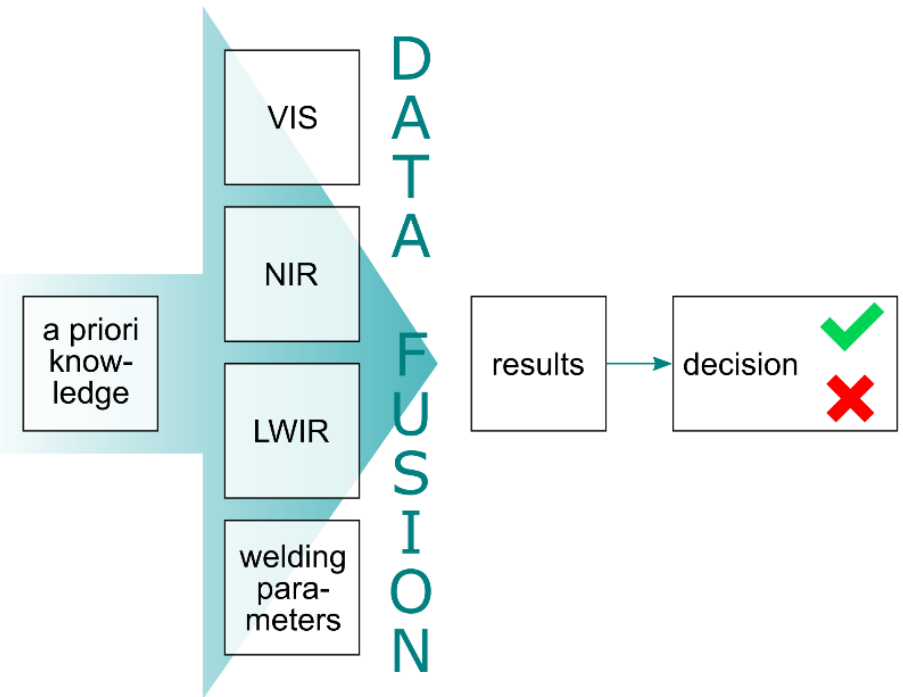

Fig. 5: Overview of the data fusion method 


\section{Experimental Results and Discussion}

The evaluation of the image processing algorithms is carried out exclusively by the manipulation of the flow rate of protective gas $Q$. The gas volume was incrementally reduced between 21 litres $/ \mathrm{min}$ and 3 litres $/ \mathrm{min}$. The main focus was on the detection of pores and the decisive limit value of the gas quantity. A flawless weld seam was achieved with the following parameters: $Q=12$ litres/min, $U=23.2 \pm 0.25 \mathrm{~V}$ and $I=193.3 \pm 4.71 \mathrm{~A}$. This weld serves as a reference for the following tests.

For the first analysis, the LWIR-images were evaluated as described in section 4.2. Fig. 6 shows the images of passive thermography of three different gas flow rates: a) $Q=12$ litres $/ \mathrm{min}$, c) $Q=9$ litres $/ \mathrm{min}$ and e) $Q=4.5$ litres $/ \mathrm{min}$. The green box marks the interesting area with the change of the emissivity and the analysis section for the images Fig. 6 b), d) and f). The red line marks the edge of the emissivity change as a function of a data-driven threshold. The curve in Fig. $6 \mathrm{~b}$ ) forms a parabolic shape in the direction of the welding event. The comparison with the other edge lines shows a clear change towards a flat curve with decreasing gas volume. This specific form is characteristic for a faulty weld seam.

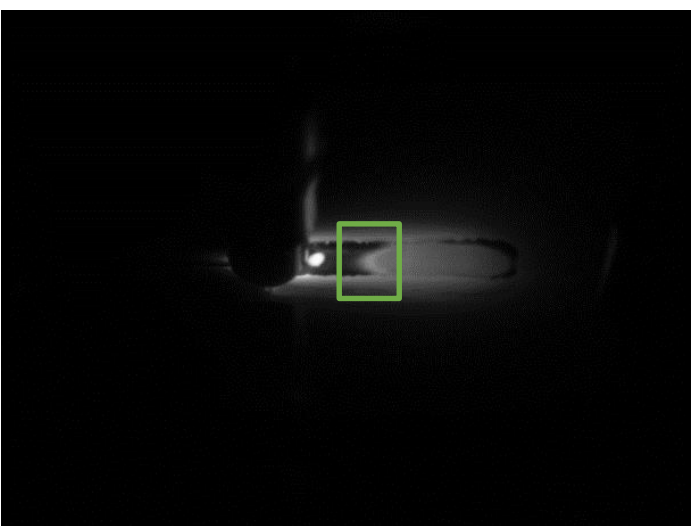

a) $Q=12$ litres $/ \mathrm{min}$

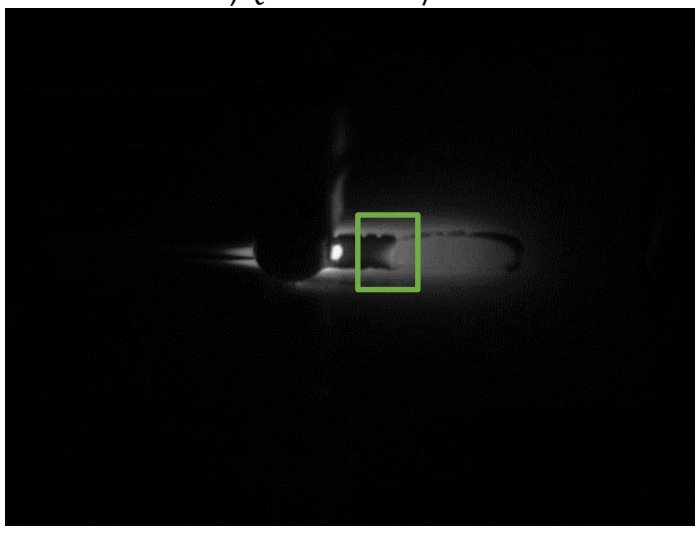

c) $Q=9$ litres $/ \mathrm{min}$

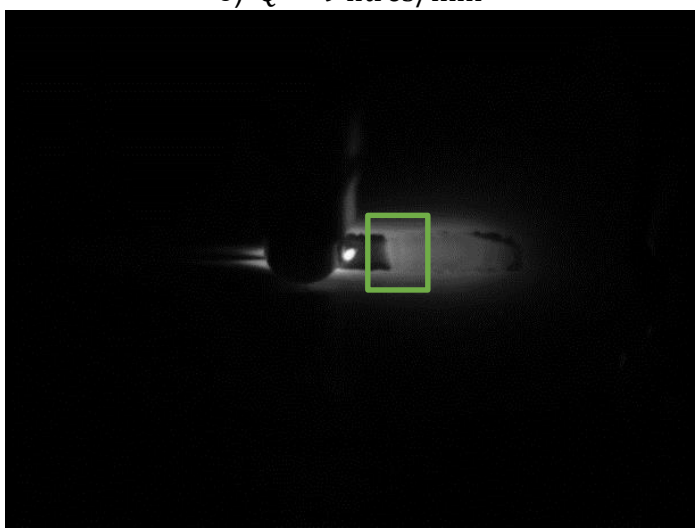

e) $Q=4.5$ litres $/ \mathrm{min}$

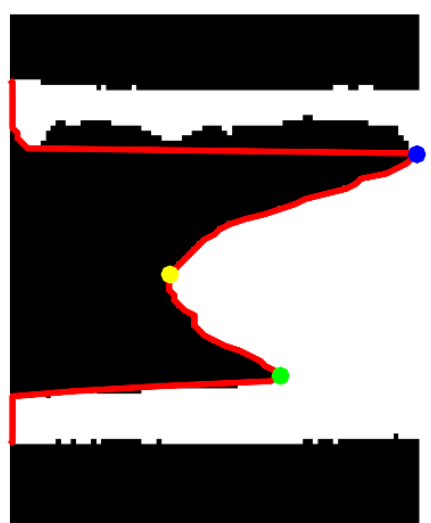

b) section of a)

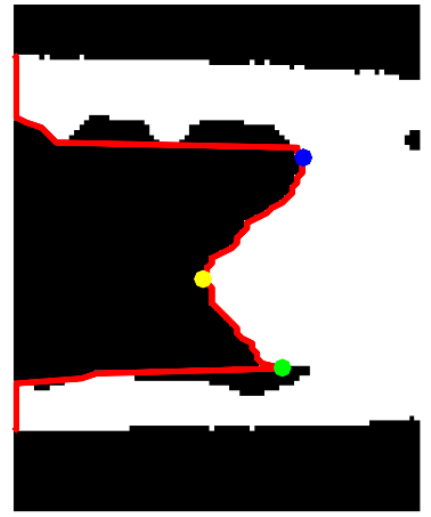

d) section of c)

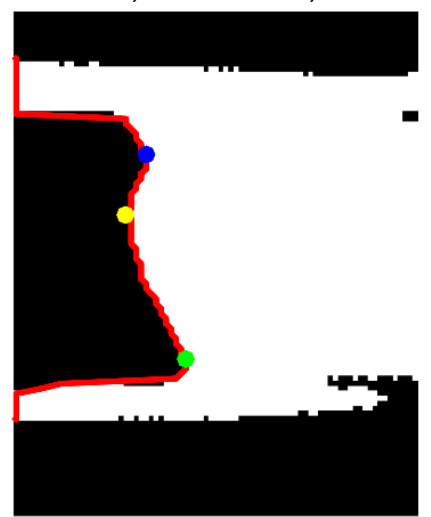

f) section of e)

Fig. 6: a), c) and e): LWIR-images change of emissivity with analysis section (green box); b), d) and f): Binary image of section with emissivity edge line (red), first two maxima $\operatorname{Max}_{1}$ and $\operatorname{Max}_{2}$ (blue, green) and minimum Min (yellow) 
According to this curve, the determination of the first two maxima ( $\operatorname{Max}_{1}$ and $\operatorname{Max}_{2}$ ) and a minimum (Min) have been implemented. Table 1 shows the calculations of the median of the equations $b_{1}, b_{2}$ and $b_{3}$ for the gas flow rates investigated. These equations represent three ways of calculating the distances between minimum and maximum. In each recorded LWIR-image, extrema are determined and used to calculate the median separate for the equations $b_{1}, b_{2}$ and $b_{3}$. The results of the welding process with a gas flow rate of 12 litres/min serve as a reference for the evaluation of the other measurements. As expected from the edge curves, each median shows a strong reduction for gas flow rates $<12$ litres/min. Based in this, it can be concluded that these measurements show possible imperfections and must be additional viewed manually. The effects of gas flow rates $>12$ litres/min cannot be clearly evaluated and must be investigated further. If analysis of other measurements shows a median different from the range of values for a gas flow rate of 12 litres/min, a more detailed examination with the other methods presented is recommended.

Table 1. Median of maxima and minimum of the emissivity change curve

\begin{tabular}{cccc}
\hline & \multicolumn{2}{c}{ median $\left(b_{n}\right)$ with $n=1,2,3$ in pixels } \\
\cline { 2 - 4 } Flow rate protective gas $Q$ & $b_{1}=$ Max $_{1}-$ Min & $b_{2}=$ Max $_{2}-$ Min & $b_{3}=\frac{\left(\text { Max }_{1}+\text { Max }_{2}\right)}{2}-$ Min \\
\hline 21 litres/min & 22 & 13 & 19 \\
18 litres/min & 32 & 9.5 & 20.75 \\
15 litres/min & 30 & 15 & 24.5 \\
12 litres/min & 40 & 25 & 33.5 \\
9 litres/min & 26 & 13 & 20.5 \\
6 litres/min & 12 & 6 & 8.5 \\
4.5 litres/min & 12 & 4 & 9 \\
\hline litres/min & 0 & 0 & 0 \\
\hline
\end{tabular}

Fig. 7 shows the cooling process of the weld seam for a few frames immediately after the end of the welding process. The lines represent the median about all wavelengths of the NIR-camera for measurements with a gas flow rate from 21 to 3 litres/min. The course of curve with a gas flow rate $\geq 12$ litres/min shows a larger heat sector area by a factor of two (frame 1) compared to the measurements with a gas flow rate of 9, 6, 4.5 and 3 liter/min. However, the cooling (fall of the course) of all weld seams shows a similar course. The measurement with a gas flow rate of 3 litres $/ \mathrm{min}$ has a smaller number of pixels representing a heat sector. Just one third of the pixels start from frame four in comparison to the measurements with a gas flow rate of 9,6 and 4.5 liter $/ \mathrm{min}$. The approach of this method is promising and needs further investigation.

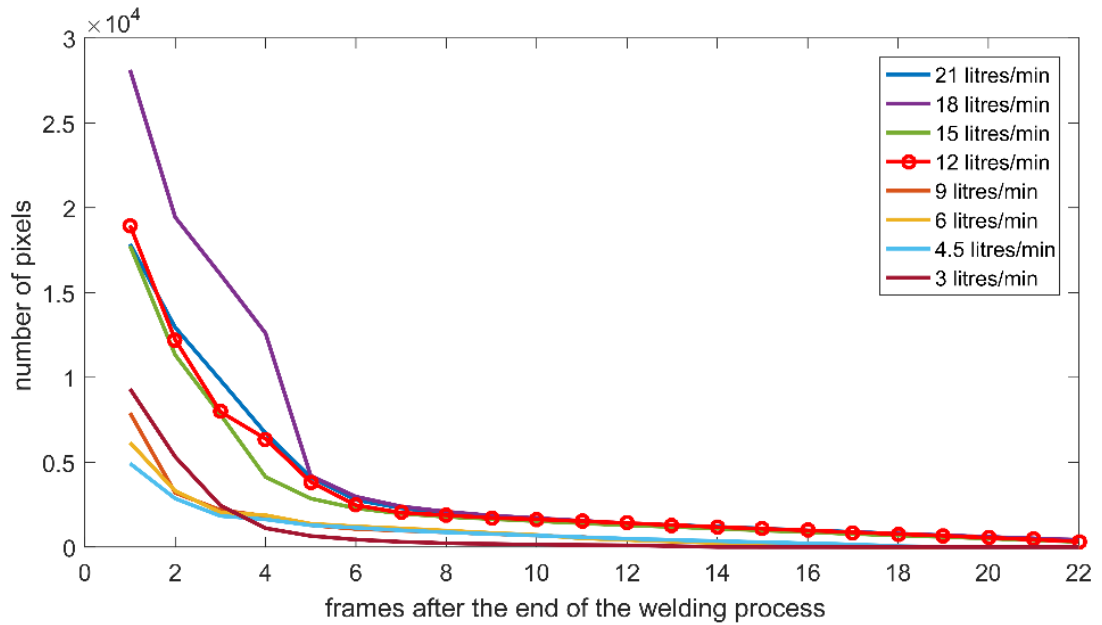

Fig. 7: Cooling process of the measurements with gas flow rates from 21 to 3 litres/min

Another method for evaluating welds is the analysis of the seam surface. The images of the visual camera are particularly important for this procedure. By applying the method described in section 4.2 , the incidences of angles occurring in the area of the weld are recorded. Fig. 8 shows these incidences in a polar plot for the gas flow rates of 12 litres/min (a) and 3 litres/min (b). For 12 litres/min, the edges of the weld seam are represented by the clearly defined course of the curve at 0 and $\pi$. However, the course is less pronounced with $\frac{\pi}{2}$ and $-\frac{\pi}{2}$. The median of the amount of defects of all recorded wavelengths is $m=0.053$ and thus below the set limit of 0.1 . This data-driven threshold has been selected based on the calculation of $\operatorname{median}(m) \approx 0.1$ (rounded down). The ratio for the measurement with 3 litres is 0.62 . The larger the ratio, the greater the probability of a faulty weld. If the deviation from the limit value increases to 1 , surface pores are to be expected. 


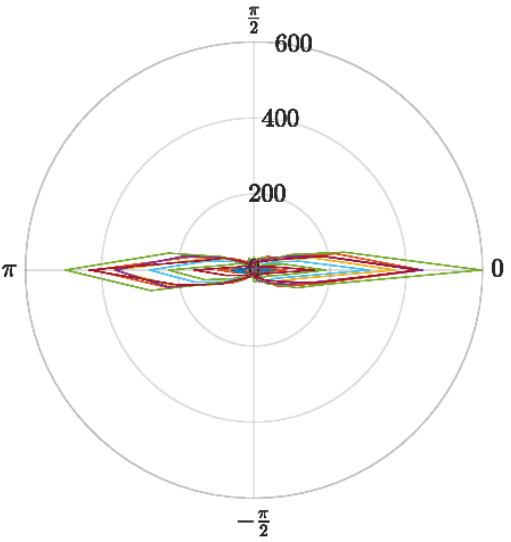

a) $Q=12$ litres $/ \mathrm{min}$

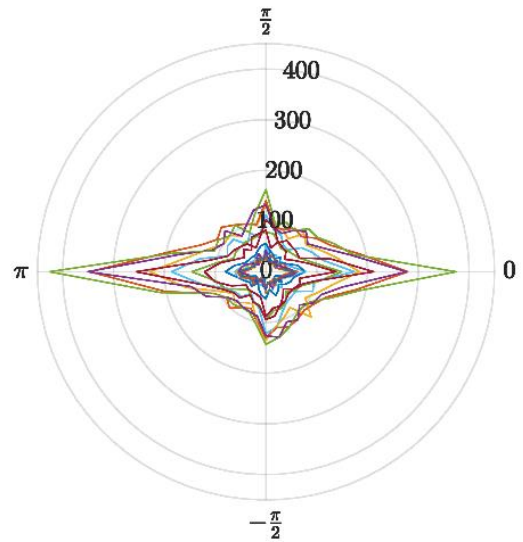

b) $Q=3$ litres/min

Fig. 8: Polar plot about incidence of angles for two different gas flow rates

Fig. 9 shows the amount of defects $a$ for each recorded wavelength in the bar plot divided into each measurement with gas flow rates from 21 to 3 litres/min. To reduce the influence of individual wavelengths and outliers, the median is calculated separately for each gas flow rate. According to the results above, the median of the measurement with 12 liters/min is the basis for determining the threshold. If this value is greater than 0.1 , this weld is considered defective. The measurement at 3 litres/min shows clearly visible surface pores (Fig. 10 e and f). Therefore, a value clearly different from the threshold is expected, which is confirmed by the value $m=0.620$. The measurement with 9,6 and 4.5 litres $/ \mathrm{min}$ also have an increased value, which indicates a faulty seam. These seams show a slight change in the surface roughness, which increases with decreasing gas volume. This dependency is quantified by using this method.

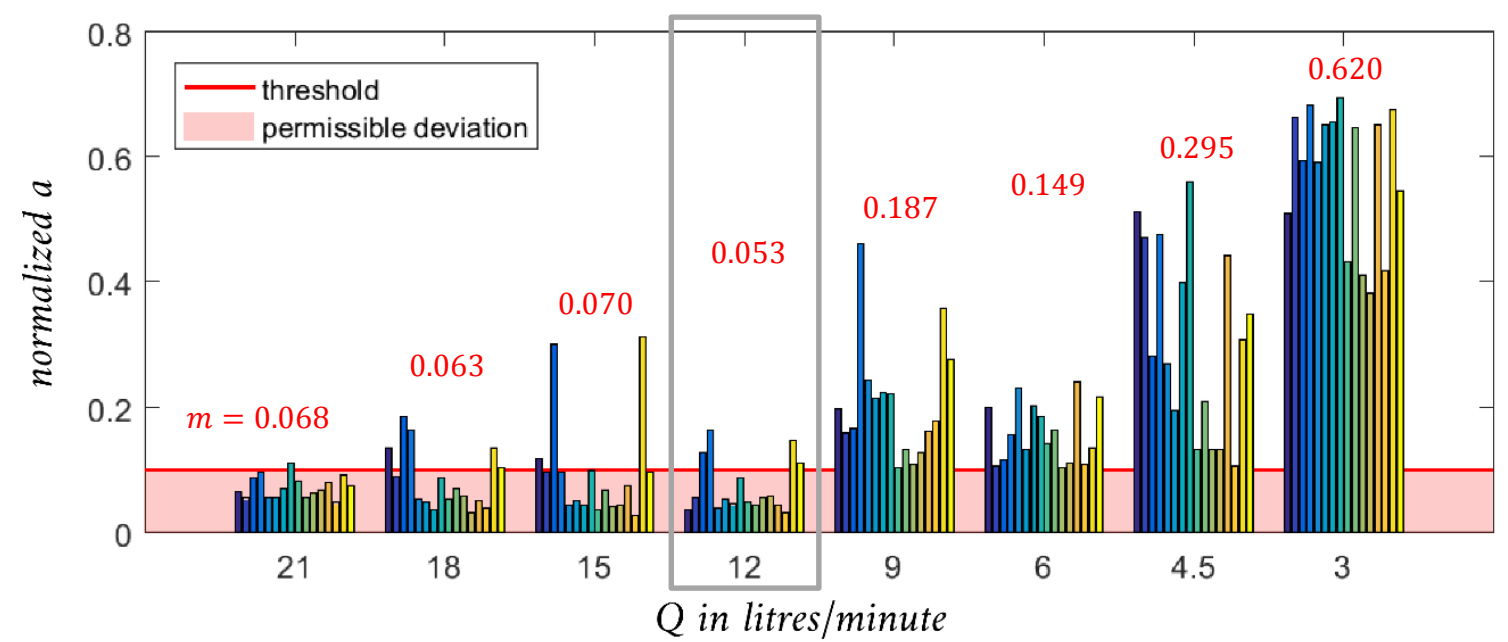

Fig. 9: Ratio bar plot represents different narrow-band frequency bands with median(a) about all wavelengths for each gas flow rate (red values); determined threshold at 0.1

According to the analysis of the VIS-images above, Fig. 10 shows pictures (left) and grindings (right) of the longitudinal sections of the weld seam with 6, 4.5 and 3 litres/min gas flow rate. The welds were made from right to left. Destructive testing was performed to verify the results of the previous non-destructive examinations. Despite almost no visible surface pores (Fig. $10 \mathrm{a}$ and $\mathrm{b}$ ), $m$ of these three measurements increases continuously.

The weld seam with 6 litres/min has some gas pores and a lot of microscopic pores at the beginning as well as a lack of fusion at the end of the longitudinal section, as shown in Fig. 10 b). Fig. $10 \mathrm{~d}$ ) shows a lot of microscopic pores all over the weld seam as well as clustered localized porosity at the beginning of the weld. These macroscopic pores can possibly lead to a stronger surface roughness of the seam. This would explain the result of the large $m=0.295$. Despite the lack of surface pores, the results from Fig. 9 suggest faulty welds for gas flow rates of $6,4.5$ and 3 litres/min. This assumption is confirmed by the results in Fig. 10. 


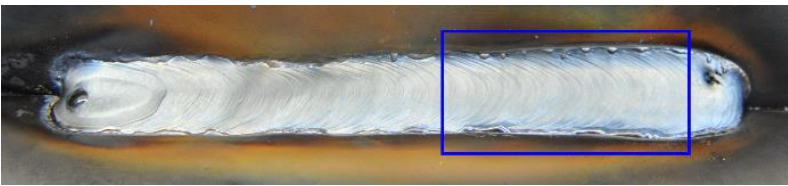

a) $Q=6$ litres $/ \mathrm{min}$

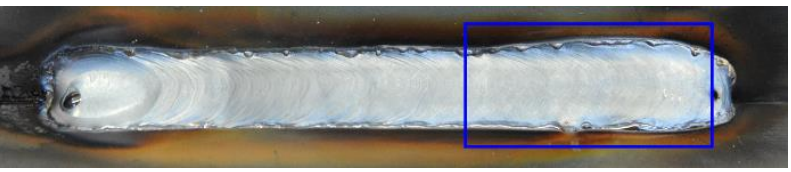

c) $Q=4.5$ litres $/ \mathrm{min}$

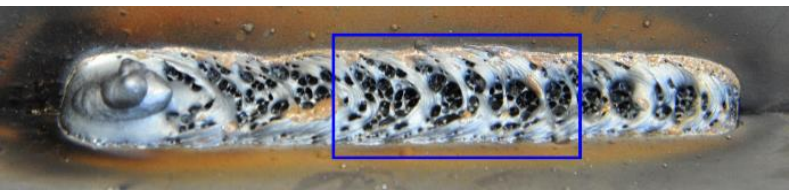

e) $Q=3$ litres $/ \mathrm{min}$

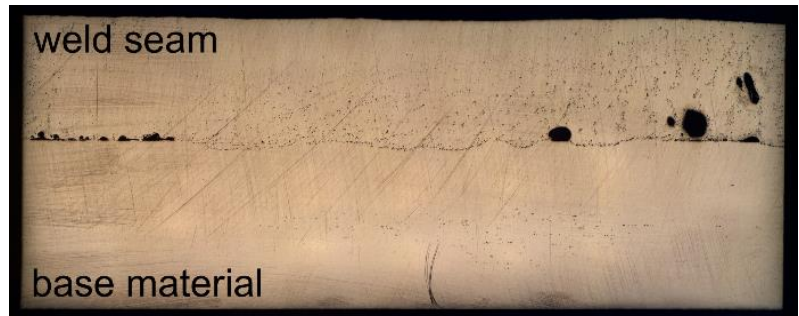

b) grinding of longitudinal section of a)

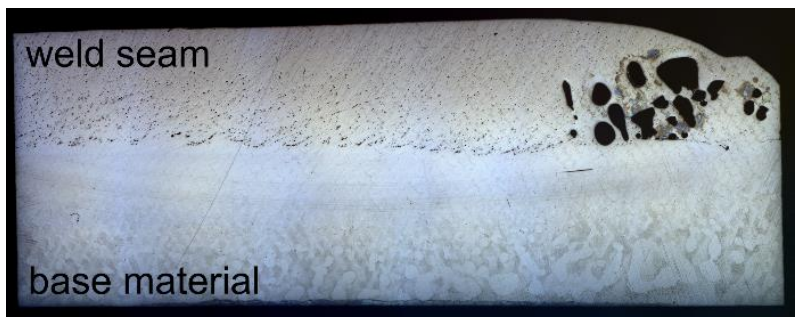

d) grinding of longitudinal section of c)

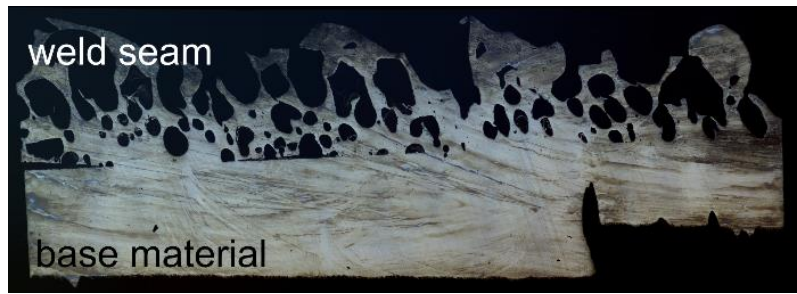

f) grinding of longitudinal section of e)

Fig. 10: a), c) and e): Pictures of weld seam with section of grinding (blue box);

b), d) and f): Grinding of longitudinal section,

During the welding process, the welding parameters current $I$ and voltage $U$ were continuously recorded. These welding parameters for the different gas flow rates are shown in box plots (Fig. 11). The median(I) deviates around the value of $197 \mathrm{~A}$, except for the last measurement with 3 litres/min (Fig. $11 \mathrm{a}$ ). Its current is $187 \mathrm{~A}$. In addition, it is subject to a strong scatter with the standard deviation of $78 \mathrm{~A}$. The welding voltage shows a comparable behaviour at the last measurement (Fig. $11 \mathrm{~b}$ ). The median $(U)$ of the remaining measurements is about $23 \mathrm{~V}$. In comparison, its voltage is also around $23 \mathrm{~V}$, but its standard deviation is approximately $8 \mathrm{~V}$. The measurements with 9,6 and 4.5 litres/min also show an increased standard deviation (1.3 V on average) compared to flautless measurement at 12 litres/min (standard deviation of $0.25 \mathrm{~V}$ ) gas flow rate.

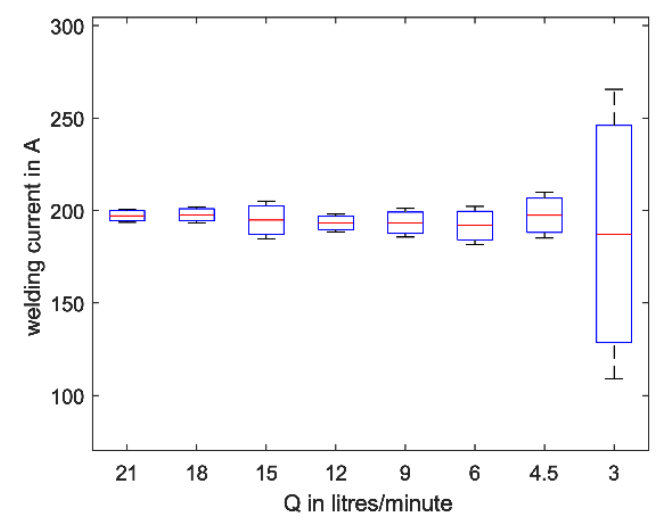

a) $\operatorname{median}(I)$ (red line)

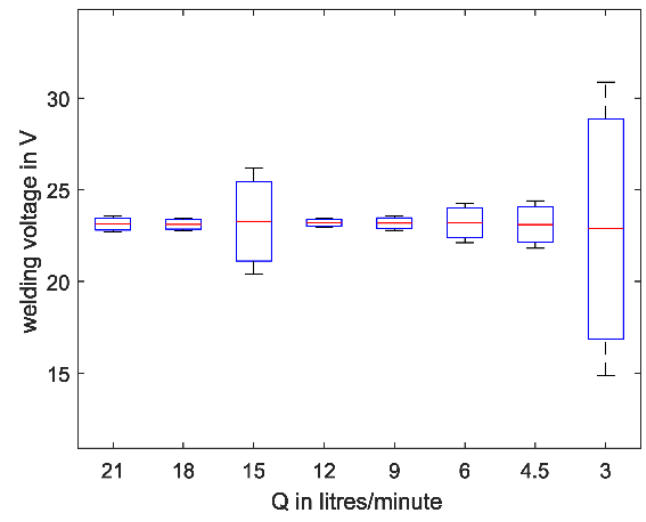

b) median $(U)$ (red line)

Fig. 11: Welding parameters current I and voltage $U$ as well as their standard deviation 


\section{Conclusion and Future Work}

In this paper, an approach for a non-destructive in-line inspection system of a fillet weld process is realized by using data fusion. The results show the performance of the connection of an image acquisition system and welding parameters as well as a priori knowledge.

The investigation of passive thermography shows that the manipulation of the gas flow rate has an influence on the shape along the edge of change in emissivity. The reduction of the flow rate results in an increasingly flat curve. The difference of prominent points (maxima and minimum) along the edge line and their median decreases significantly. The lower the median, the more likely there is a faulty weld. The investigation of the cooling process shows that the near infrared spectral range contributes to the evaluation of the measurements. However, further investigations are necessary for detailed results. By calculating the amount of defects $a$ of the sums $p_{v}$ and $p_{h}$, a statement can be made about scaliness up to porosity of the weld seam. These fine surface changes could be overlooked in a classic visual inspection. Thus, this method serves to detect previously unnoticed imperfections. Additionally, the results of the analysis of the welding parameters support the assumption that, not only the measurement with 3 litres/min also the measurements with 9,6 and 4.5 litres/min contain imperfections. The grindings support the results of non-destructive testing, which were achieved by means of using camera systems and digital image processing. The influence of wavelengths on these methods and the interpretation of the results of the measurements at 21,18 and 15 litres/min has to be investigated.

The overall result shows, that the weld seam with $3 \mathrm{litres} / \mathrm{min}$ is a faulty seam. This result also indicates that the seams with 9, 6 and 4.5 litres/min must additional be checked manually. By applying data fusion, the results of the individual investigations are summarized and provide an overall result with greater significance. The application of such a system in an automated manufacturing process can help to detect defects early and adjust or stop the process, respectively.

\section{Acknowledgement}

This work has been funded by the Federal Ministry of Education and Research (BMBF) in the project "Multi-modal data acquisition and analysis" (03PSIPT3A, project acronym 3dStahl).

SPONSORED BY THE

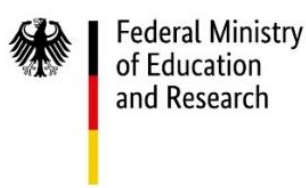

\section{REFERENCES}

[1] Blackbird Robotersysteme $\mathrm{GmbH}$, Dynamische Nahtverfolgung. [Online] Available: https://www.blackbirdrobotics.de/produkte-loesungen/dynamische-nahtverfolgung.html. Accessed on: Nov. 20, 2017.

[2] P. Sievi, "Verfahren zur Erhöhung der Genauigkeit der Führung des Bearbeitungslasers von Fügevorrichtungen" DE102010060162B3.

[3] VITRONIC Bildverarbeitungssysteme $\mathrm{GmbH}$, Vollautomatische 3D-Schweißnahtprüfung und Lötnahtprüfung mit VIRO WSI. [Online] Available: https://www.vitronic.de/industrie-undlogistikautomation/branchen/automotive/schweissnahtpruefung.html. Accessed on: Nov. 20, 2017.

[4] V. Schauder, M. Häussler, M. Kiese, and O. Hollmann, "Vorrichtung zur Bewertung der Qualität von Schweissnähten durch Erfassung des Temperaturprofils der sich abkühlenden Schmelze während des Schweissens" EP000001944119B1.

[5] J. N. Pires et al., "Welding robots," IEEE Robotics \& Automation Magazine, vol. 10, no. 2, pp. 45-55, 2003.

[6] XIMEA GmbH, "XiSpec Brochure," 2016.

[7] InfraTec $\mathrm{GmbH}$, "VarioCAM® HD head: Thermographic Solution for Use in Industry and Research," 2016.

[8] B. Corves, L. Deters, P. Dietz, and K. Dilger, "Bauteilverbindungen," in Taschenbuch für den Maschinenbau: Mit Tabellen, H. Dubbel, K.-H. Grote, J. Feldhusen, P. Dietz, and G. Ziegmann, Eds., 22nd ed., Berlin: Springer, 2007.

[9] K. Simmen, B. Buch, A. Breitbarth, and G. Notni, "Multimodal Image Acquisition and Processing System for In-line Quality Monitoring of Welding Processes," 2017.

[10] B. Jähne, Digitale Bildverarbeitung und Bildgewinnung, 7th ed. Berlin, Heidelberg: Springer, 2012.

[11] Richard Hartley and Andrew Zisserman, Multiple View Geometry in Computer Vision: Cambridge University Press, 2003.

[12] M. Correns, "Subpixelgenaue Kantenortsbestimmung in digitalen Mehrkanalbildern, dargestellt am Beispiel von Sensoren mit Bayer Pattern Color Filter Array," Dissertation, Technische Universität IImenau, IImenau, 2011. 